\title{
A Bipartite Recombinant Yeast System for the Identification of Subtype-Selective Estrogen Receptor Ligands
}

Kaiwei Liang $\cdot$ Liuqing Yang $\cdot$ Zhimin Xiao ·

Jian Huang

Published online: 6 January 2009

(C) Humana Press 2008

Erratum to: Mol Biotechnol (2009) 41:53-62

DOI 10.1007/s12033-008-9097-9

In the original article, the name of the University was omitted from the authors' affiliation. The correct affiliation is as follows: State Key Laboratory of Virology, College of Life Sciences, Wuhan University.

The online version of the original article can be found under doi:10.1007/s12033-008-9097-9.

K. Liang $\cdot$ L. Yang $\cdot$ Z. Xiao $\cdot$ J. Huang $(\bowtie)$

State Key Laboratory of Virology, College of Life Sciences,

Wuhan University, Wuhan, Hubei Province 430072, China

e-mail: jianhuang@whu.edu.cn 